\title{
Correlación entre diagnóstico ecográfico e histopatológico de poliposis vesicular en la Clínica Good Hope 2008-2014
}

\author{
Emiliano Contreras Castro ${ }^{1}$, Paul Alfaro Fernández ${ }^{2}$, Flor Contreras Castro ${ }^{3}$, Roberto Luna Victoria ${ }^{4}$, Irma Contreras Alomía ${ }^{5}$
}

\section{RESUMEN}

Objetivo: Determinar la correlación entre el diagnóstico ecográfico y el resultado histopatológico de poliposis vesicular en los pacientes y encontrar el valor predictivo positivo de la ecografía en el diagnóstico de esta patología.

Material y Métodos: Es una investigación no experimental, transversal, descriptiva y correlacional, realizada en una muestra de 128 pacientes operados con diagnóstico de poliposis vesicular en la clínica Good Hope entre 2008 y 2014 . Se revisaron los informes ecográficos e histopatológicos de estos pacientes y se buscó la relación mediante el coeficiente de correlación de Spearman.

Resultados: El 67,2\% de la muestra correspondió al sexo femenino y el 32,8\% al sexo masculino; la edad promedio fue de 43,4 años. El 74,2\% de los 128 casos presentó pólipos según el examen histopatológico, de los cuales el 94,7\% fueron pseudopólipos y $84 \%$ fueron pólipos de colesterol; solo el 5,3\% de los pólipos fueron verdaderos (adenomas) y ninguno de ellos fue maligno, el pólipo más grande midió $13 \mathrm{~mm}$. El valor predictivo positivo de la ecografía en el diagnóstico de poliposis vesicular fue $74.21 \%$. La correlación entre el número de pólipos por ecografía e histopatología según el coeficiente de Spearman fue muy baja, directa y significativa ( $R h o=0,189, p=0,032$ ).

Conclusión: Existe una correlación directa entre el diagnóstico ecográfico y el resultado histopatológico de poliposis vesicular; la ecografía puede considerarse como un método confiable para el diagnóstico de esta patología.

Palabras clave: pólipo vesicular, ecografía, estudio histopatológico.

\section{Correlation between sonographic diagnosis and histopathological results of gallbladder poliposis in Good Hope Clinic 2008-2014}

\begin{abstract}
Objective: To determine the correlation between sonographic diagnosis and histopathological results of gallbladder polyposis and find the positive predictive value of ultrasound in the diagnosis of this pathology.

Material and Methods: It is a non experimental, transversal, descriptive and correlational study with a sample composed of all patients operated with a diagnosis of gallbladder polyps in the Good Hope Clinic between the years 2008 and 2014. A total of 128 patients were observed. Histopathological and sonographic reports of these patients were reviewed and the statistical correlation of both studies was sought by the test of Spearman.

Results: Reveals that $67,2 \%$ were females and $32,8 \%$ were males; the average age was 43,4 years; $74,2 \%$ presented polyps by histopathological examination, of which $94,7 \%$ were pseudopolyps, with $82 \%$ cases of cholesterolpolyps, only $5,3 \%$ were true polyps (adenomas) and none of them were malignant. The positive predictive value of ultrasound in the diagnosis of gallbladder polyposis was $74,21 \%$. According to the Spearman coefficient the correlation between the number of polyps by ultrasonography and histopathology was low, direct and significant ( $R h o=0,189 ; p=0,032$ ).

Conclusions: We conclude that there is a correlation between the ultrasound diagnosis and histopathological result of gallbladder polyps and ultrasound can be considered a reliable method for the diagnosis of gallbladder polyps.
\end{abstract}

Key words: gallbladder polyp, ultrasonography, histopathological study.

1. Jefe de Cirugía de la Clínica Good Hope.

2. Médico Epidemiólogo, docente de la USMP.

3. Doctora en Salud Pública, coordinadora de Posgrado de la UPeU.

4. Jefe de archivo de la Clínica Good Hope.

5. Licenciada especialista en Centro Quirúrgico de la Clínica Good Hope. 


\section{INTRODUCCIÓN}

Los pólipos de la vesícula biliar son proyecciones de la mucosa hacia el lumen de este órgano que pueden deberse a depósitos lipídicos, procesos inflamatorios o neoplasias (1).Se clasifican en lesiones malignas (adenocarcinomas, metástasis y otras) y benignas, que pueden ser tumorales (adenoma, leiomioma, lipoma) o pseudotumorales (pólipos de colesterol, inflamatorios, hiperplásicos, adenomiomas, otros) (2). Por ser el cáncer de vesícula biliar uno de los de peor pronóstico entre los cánceres del tracto digestivo (3), se plantea la necesidad de extirpar la vesícula biliar en los pacientes con diagnóstico ecográfico de poliposis vesicular como una medida preventiva, pues existe un eventual riesgo de malignización (4-6). La secuencia pólipo-carcinoma de vesícula biliar como en los carcinomas colorrectales, ha sido propuesta como teoría carcinogénica del cáncer de la vesícula biliar $(7,8)$, aunque estudios moleculares sugieren que la secuencia adenoma-carcinoma no se encuentra involucrada en la mayoría de los cánceres vesiculares $(9,10)$.

En estudios realizados, la prevalencia de poliposis vesicular varía entre $4-7 \%$ de la población adulta que se realiza ultrasonido abdominal $(11,12)$. Esta patología es todavía un problema para el cirujano general, pues no existen guías clínicas ni estudios basados en la evidencia para el manejo de los pólipos vesiculares (13). La duda consiste en determinar cuándo se debe intervenir a una persona con este diagnóstico, pues hay un porcentaje de casos falsos positivos y otros, donde más del $70 \%$ corresponde a lesiones pseudotumorales $(14,15)$. Sobre este punto hay controversia; sin embargo, la mayoría de los estudiosos del tema recomiendan extirpar la vesícula biliar cuando el pólipo mide más de $10 \mathrm{~mm}$, pues el riesgo de malignidad aumenta con el tamaño de la lesión(11).

En los estudios revisados el diagnóstico de poliposis vesicular se basó en la ecografía, por lo que se planteó como objetivo general determinar la correlación entre diagnóstico ecográfico e histopatológico de poliposis vesicular en los pacientes atendidos en la clínica Good Hope; y, como objetivos específicos: identificar sus características sociodemográficas, describir los resultados histopatológicos y determinar el valor predictivo positivo de la ecografía en el diagnóstico de poliposis vesicular.

\section{MATERIAL Y MÉTODOS}

Es un estudio no experimental, cuantitativo, observacional y descriptivo, se basa en el método estadístico para la prueba de hipótesis y presentación de resultados; en función a los objetivos que persigue es una investigación básica (16), transversal por que la medición se realiza en un momento dado (17), retrospectivo y de serie de casos porque se basa en la identificación y descripción de los casos de pólipos de vesícula biliar que fueron atendidos en los últimos siete años.

Ingresaron al estudio la serie de casos de todos los pacientes con diagnóstico ecográfico de poliposis vesicular a quienes luego de la cirugía se les realizó el estudio histopatológico, el periodo de los casos incluidos corresponden a los casos atendidos del 2008 al 2014. La finalidad de la inclusión de estos pacientes fue medir la correlación entre las dos pruebas diagnósticas y el valor predictivo positivo de la ecografía.

El diseño se representa en el siguiente gráfico:

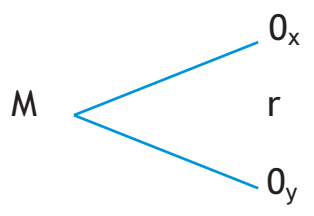

Dónde:

M: Muestra

$0_{x}$ : Observación de la variable diagnóstico ecográfico

$0_{y}$ : Observación de la variable resultado histopatológico

r: Coeficiente de correlación

La población universo son todos los casos operados desde el 2008 al 2014 con diagnóstico ecográfico de poliposis vesicular, en total de 173 casos.

La selección de la muestra se realizó atendiendo a los criterios de limpieza de datos que significó la exclusión de todos los casos en cuyas historias clínicas no constaba el informe ecográfico y/o histopatológico, con la finalidad de evitar alteraciones al momento de determinarla relación entre ambas variables. Se incluyeron en el estudio todos los casos cuyos informes ecográficos e histológicos estuvieran consignados en sus respectivas historias clínicas, resultando el tamaño final de la muestra 128 casos. En cuanto a la prueba de 
hipótesis de relación entre variables, se utilizó el coeficiente de correlación de Spearman debido a que la distribución de los datos no cumplieron con los supuestos de normalidad para el indicador: cantidad de pólipos en el diagnóstico ecográfico y el resultado histopatológico.

\section{RESULTADOS}

Tabla 1. Características de los pacientes operados por poliposis vesicular

\begin{tabular}{|c|c|c|}
\hline Características & n & $\%$ \\
\hline \multicolumn{3}{|l|}{ Sexo } \\
\hline Masculino & 42 & 32,8 \\
\hline Femenino & 86 & 67,2 \\
\hline \multicolumn{3}{|c|}{ Grupo etario por etapas de desarrollo } \\
\hline Adolescente & 2 & 1,6 \\
\hline Adulto Joven & 61 & 47,7 \\
\hline Adulto Maduro & 49 & 38,3 \\
\hline Adulto Mayor & 16 & 12,5 \\
\hline
\end{tabular}

Nota: $\operatorname{Edad}(\bar{x}=43,35 ; S=14,93 ; M e=39 ; M i n=16, \operatorname{Max}=79)$

La tabla 1, muestra que el $32,8 \%$ de las historias clínicas seleccionadas corresponde a pacientes del sexo masculino y el $67,2 \%$ al sexo femenino. El $1,6 \%$ fue adolescente, el $12,5 \%$ adulto mayor, al 38,3 adulto maduro y el $47,7 \%$ adulto joven (18).

Tabla 2. Resultados histopatológicos de los pacientes operados por poliposis vesicular

\begin{tabular}{|lrc|}
\hline Resultados histopatológicos & $\mathrm{n}$ & $\%$ \\
\hline Tumoral & & \\
\hline Adenoma & 5 & 5,3 \\
\hline Pseudotumoral & 89 & 94,7 \\
\hline Pólipo de colesterol & 79 & 84,0 \\
\hline Adenomiosis & 2 & 2,1 \\
\hline Pólipos Inflamatorios & 1 & 1,1 \\
\hline Pólipos hiperplásicos & 3 & 3,2 \\
\hline Pólipos de colesterol y adenomiosis & 2 & 2,1 \\
\hline Pólipos de colesterol e hiperplásico & 1 & 1,1 \\
\hline Adenomiosis y pólipo hiperplásico & 1 & 1,1 \\
\hline Total & 94 & 100 \\
\hline
\end{tabular}

Nota: Cantidad de pólipos ( $\overline{\mathrm{X}}=3,68 ; \mathrm{S}=3,08 ; \operatorname{Min}=1, \operatorname{Max}=18)$; Tamaño de pólipo (Min=1, Max=13); 34 casos no presentaron pólipos.

La tabla 2, muestra que de las 128 historias estudiadas en 5,3\% de ellas se encontró tumor adenomatoso, en $94,7 \%$ pseudotumores distribuidos de la siguiente forma: $84 \%$ presentaron pólipos de colesterol; $2,1 \%$ adenomiosis; $1.1 \%$ pólipos inflamatorios, 3,2\% pólipos hiperplásicos; $2,1 \%$ pólipos de colesterol y adenomiosis, 1,1\% pólipo de colesterol e hiperplásico, y 1,1\% adenomiosis y pólipo hiperplásico. La cantidad de pólipos mínima encontrada fue de 1 y la cantidad máxima de pólipos encontrados fue de 18; el tamaño mínimo de pólipo encontrado fue de $1 \mathrm{~mm}$ y el tamaño máximo de $13 \mathrm{~mm}$. 
Tabla 3. Valor predictivo positivo de la ecografía en el diagnóstico de poliposis vesicular

\begin{tabular}{|l|c|c|c|c|}
\hline & \multicolumn{3}{|c|}{ Resultado histopatológico } \\
\hline Diagnóstico ecográfico & \multicolumn{2}{|c|}{ Sí No } \\
\hline Positivo & $\mathrm{n}$ & $\%$ & $\mathrm{n}$ & $\%$ \\
\hline Negativo & 94 & 73,4 & 34 & 26,6 \\
\hline
\end{tabular}

La tabla 3, muestra que de los 128 casos atendidos, 94 de ellos fueron verdaderos positivos y 34 falsos positivos, el valor predictivo positivo de la ecografía es de $73.4 \%$

Tabla 4. Correlación entre el diagnóstico ecográfico y los resultados histopatológicos por número de pólipos encontrados

\begin{tabular}{|c|c|c|c|}
\hline & \multicolumn{3}{|c|}{ Resultado histopatológico } \\
\hline Diagnóstico ecográfico & $n$ & Rho & $\mathrm{p}$ \\
\hline
\end{tabular}

La tabla 4, muestra los resultados del análisis de relación a través del coeficiente de correlación de Spearman entre la cantidad de pólipos determinados por ecografía y la cantidad de pólipos reportados por el informe histopatológico, evidenciándose que existe una relación muy baja directa y significativa entre las cantidades especificadas por cada una de ellas.

\section{DISCUSIÓN}

El presente estudio sobre poliposis vesicular se desarrolló debido a la prevalencia de esta patología referida en diversos estudios, la cual oscila entre 4 y 7 $\%$ de la población adulta $(11,12)$ y el número considerable de casos atendidos por poliposis vesicular en la clínica Good Hope. La manifestación de esta patología reportada en esta investigación fue mayor en pacientes del sexo femenino con un $67,2 \%$, coincidiendo con los resultados de estudios realizados en otros países (19-21), lo que corrobora que el sexo femenino es un factor de riesgo para el desarrollo de esta patología, pudiendo llegar a estadios avanzados de malignidad(22).

Del mismo modo, la edad también es considerada como factor de riesgo para el desarrollo de los pólipos, ya que la mayor incidencia se da a los 65 años, aunque últimos estudios refieren que se presenta en personas de la cuarta y quinta década de vida (23), otros estudios reportan promedios de 40,44 y 48,7 años $(4,19,24)$ coincidiendo con los resultados de este estudio donde se encontró una edad promedio de 43,4 $\pm 14,9$ años; sin embargo, es importante mencionar que existe un porcentaje considerable de adultos jóvenes que presentaron esta patología, considerándose importante investigar las causas de la enfermedad en este grupo etario.
De los 94 casos $(73,4 \%)$ en los cuales se encontró pólipos en el examen histopatológico, 89 de ellos $(94,7 \%)$ fueron pseudopólipos, con $79(84 \%)$ casos de pólipos de colesterol, lo que concuerda con los hallazgos de estudios realizados en España donde del total de 30 pacientes, 20 reportaron pseudopólipos (13), en Perú el 95,4\% correspondió a pólipos colesterolósicos (25) y diversos estudios en Chile reportan $67,9 \%$ pólipos de colesterol (24), 95,7\% pseudotumores compuestos (19) y $100 \%$ pólipos de colesterol (4). Esto significa que la mayoría de los pólipos no son malignos; sin embargo, debido a la agresividad del cáncer de vesícula biliar (3) es importante considerar las características de los pólipos en el estudio ecográfico y practicar el estudio histopatológico en todos los pacientes colecistectomizados por pólipos para su tratamiento oportuno. La razón por la que en este estudio no se encontró pólipos malignos, puede ser explicado por el tamaño de los pólipos encontrados que fue menor de $13 \mathrm{~mm}$; esto confirmaría lo que sustentan los estudios analizados los cuales coinciden que a mayor tamaño de los pólipos mayor riesgo de malignidad $(13,19,25-28)$.

Con respecto al valor predictivo de la ecografía en el diagnóstico de poliposis vesicular se puede afirmar que el $73,4 \%$ es un buen valor para considerarse como una prueba confiable para detectar pólipos en la persona; es también importante mencionar que este porcentaje es superior al reportado en otros estudios respecto al valor predictivo de la ecografía, como es el caso del estudio realizado en los periodos 1996 al 2007 donde se encontró que de un total de 417 casos atendidos sólo el $64 \%$ presentó pólipos de vesícula en el estudio histopatológico (29). El detectar pólipos en 73 o 74 personas de cada 100 casos atendidos, indica la importancia de seguir utilizando este método diagnóstico, pues la mayoría de las instituciones de 
Correlación entre diagnóstico ecográfico e histopatológico de poliposis vesicular en la Clínica Good Hope 2008-2014

salud cuenta con un equipo ecográfico, por la accesibilidad en cuanto a costos y por ser un procedimiento no invasivo. Además, la ecografía permite visualizar la pared vesicular y el seguimiento de los pólipos en cuanto al tamaño que son aspectos básicos para realizar colecistectomía profiláctica(30).

En cuanto al análisis de correlación del número de pólipos especificados en el diagnóstico ecográfico e histopatológico del presente estudio, se encontró una relación muy baja directa y significativa $(\mathrm{Rho}=0.189$, $\mathrm{p}=0.032$ ), dicho valor en cuanto a la intensidad de la correlación es muy cercano al reportado por Lara, quien encontró un coeficiente $r=0.21$, aun cuando el $p=0.153$ en este grupo de historias analizadas indica que no existe evidencia suficiente para afirmar la correlación significativa del número de pólipos encontrados por ecografía y por histología (31). Esta situación puede ser explicada por la dificultad que existe para identificar adecuadamente el número de pólipos a través de la ecografía, ya que la ubicación de éstos o el estar superpuestos uno con otro, puede crear confusión al visualizar la cantidad real de pólipos, lo que puede generar muchas veces resultados como los encontrados por Rodríguez quien al estudiar la correlación entre el número de pólipos vesiculares diagnosticados por ecografía abdominal y los informados por anatomía patológica indican una relación inversa $(r=-0,028)(32)$.

En conclusión, la mayoría de pacientes colecistectomizados por poliposis vesicular son de sexo femenino $(67,2 \%)$; la edad promedio es de 43,4 $\pm 14,9$ años, considerándose al sexo femenino un factor de riesgo en cuanto a la presencia de pólipos vesiculares, y en nuestro estudio la mayor concentración de esta patología estuvo en el grupo etario adulto joven.

En la mayoría de los casos los pólipos fueron pseudotumorales $(94.7 \%)$ siendo los de colesterol (84\%) los más frecuentes. No se encontró signos de malignidad en ningún caso, lo que se explica porque todos los pólipos encontrados tuvieron un tamaño menor a $13 \mathrm{~mm}$.

El valor predictivo positivo de la ecografía en el diagnóstico de poliposis vesicular es de 73,4 ; lo que determina que es un método confiable en el diagnóstico y seguimiento de esta patología.

Existe correlación muy baja directa y significativa entre el diagnóstico ecográfico y los resultados histopatológicos de poliposis vesicular en pacientes colecistectomizados.

\section{REFERENCIAS BIBLIOGRÁFICAS}

1. Lee KF, Wong J, Li JCM, Lai PBS. Polypoid lesions of the gallbladder. Am J Surg [Internet]. 2004 Aug [citado 2015 Jul 7];188(2):18690. Disponible en: http://www.ncbi. nlm.nih.gov/pubmed/15249249

2. Christensen AH, Ishak KG. Benign tumors and pseudotumors of the gallbladder. Report of 180 cases. Arch Pathol [Internet]. 1970 Nov [citado 2015 Jul 7];90(5):42332. Disponible en: http://www.ncbi.nlm.nih.gov/ pubmed/4319984

3. Pejić MA, Milić DJ. [Surgical treatment of polypoid lesions of gallbladder]. Srp Arh Celok Lek [Internet]. 2003 Jan [citado $2015 \mathrm{Jul}$ 7];131(7-8):31924. Disponible en: http://www.ncbi.nlm.nih.gov/pubmed/14692147

4. Larrachea P, Escalona A, Sarras E. Pólipo vesicular. Rev Chil Cirugía [Internet]. 2003;55(2):1713. Disponible en: http://www.imbiomed.com.mx/1/1/articulos.php?method =showDetail\&id_articulo=41971\&id_seccion=2692\&id_ejem plar $=4281$ \&id revista $=163$

5. Feldman M, Friedman L, Brandt L. Sleisenger and Fordtrans Gastrointestinal and Liver Disease. 9th ed. USA: Elsevier Inc.; 2010. 1-2480 p.

6. Venturelli M F, Cárcamo I C, Born G M, Venegas O L, Bertrán V $M$, Venturelli $L A$, et al. Cáncer vesicular en colecistectomías: Análisis de sobrevida y factores pronósticos. Rev Chil cirugía [Internet]. Sociedad de Cirujanos de Chile; 2008 Aug [citado 2015 Jul 7];60(4):27781. Disponible en: http://www.scielo.cl/ scielo.php?script=sci_arttext\&pid=S0718-40262008000 400003\&lng=es\&nrm=iso\&tlng=es

7. Aldridge $M C$, Bismuth $\mathrm{H}$. Gallbladder cancer: The polypcancer sequence. Br J Surg [Internet]. 1990 Apr [citado 2015 Jul 7];77(4):3634. Disponible en: http://doi.wiley.com/ $10.1002 /$ bjs. 1800770403

8. Kozuka S, Tsubone N, Yasui A, Hachisuka K. Relation of adenoma to carcinoma in the gallbladder. Cancer [Internet]. 1982 Nov 15 [citado 2015 Jul 7];50(10):222634. Disponible en: http://www.ncbi.nlm.nih.gov/pubmed/7127263

9. Ajiki T, Fujimori T, Onoyama H, Yamamoto M, Kitazawa S, Maeda $\mathrm{S}$, et al. K-ras gene mutation in gall bladder carcinomas and dysplasia. Gut [Internet]. 1996 Mar [citado 2015 Jul 7];38(3):4269. Disponible en: http://www. pubmedcentral.nih.gov/articlerender.fcgi?artid=1383074\&t ool=pmcentrez\&rendertype=abstract

10. Roa E I, Muñoz N S, Ibacache S G, de Aretxabala U X. Desde la displasia hasta el cáncer de la vesícula biliar. Rev Med Chil [Internet]. Sociedad Médica de Santiago; 2009 Jul [citado 2015 Jun 23];137(7):87380. Disponible en: http: / / www.scielo.cl/scielo.php?script=sci_arttext\&pid=S0 034-98872009000700002\&lng=es\&nrm=iso\&tlng=es

11. Koga A, Watanabe K, Fukuyama T, Takiguchi S, Nakayama F. Diagnosis and operative indications for polypoid lesions of the gallbladder. Arch Surg [Internet]. 1988 Jan [citado 2015 Jul 7];123(1):269. Disponible en: http://www.ncbi. nlm.nih.gov/pubmed/3276295

12. Monge DZ, Monge RB, Ovares CU. Pólipos de la vesícula biliar. Rev Médica Costa Rica y Centroamérica [Internet]. 2013 [citado 2015 Jul 7];70(605):137. Disponible en: http:// new. medigraphic.com/cgi-bin/resumen.cgi?IDREVISTA= 219\&IDARTICULO=40646\&IDPUBLICACION $=4263$

13. Morera-Ocón FJ, Ballestín-Vicente J, Calatayud-Blas AM, de Tursi-Rispoli LC, Bernal-Sprekelsen JC. [Surgical indications in gallbladder polyps]. Cirugía española [Internet]. Elsevier; 2013 May 1 [citado 2015 Jul 7];91(5):32430. Disponible en: http: / / www.elsevier.es/es-revista-cirugia-espanola-36articulo-indicaciones-quirurgicas-los-polipos-vesicula90200176 
14. Colecchia A, Larocca A, Scaioli E, Bacchi-Reggiani ML, Di Biase AR, Azzaroli F, et al. Natural history of small gallbladder polyps is benign: evidence from a clinical and pathogenetic study. Am J Gastroenterol [Internet]. 2009 Mar [citado 2015 Jul 7];104(3):6249. Disponible en: http://www.ncbi.nlm.nih.gov/pubmed/19209165

15. Cortés P. Pólipos vesiculares / barro biliar: ¿Qué hacer? Gastroenterol Latinoam [Internet]. 2006;17(2):2737. Disponible en: http://myslide.es/documents/barrobiliar.html

16. Córdova I. El proyecto de investigación cuantitativa. Lima: San Marcos; 2013. 215 p.

17. Hernández R, Fernández C, Baptista P. Metodología de la investigación. 5 th ed. México: Mc GrGraw-Hill interamericana; 2010.614 p.

18. Papalia D, Wendkos S, Duskin R. Desarrollo Humano. Colombia: Mc Graw-Hill; 2000.

19. Escalona PA, León G F, Bellolio R F, Pimentel M F, Guajardo B $M$, Gennero R, et al. Pólipos vesiculares: correlación entre hallazgos ecográficos e histopatológicos. Rev Med Chil [Internet]. 2006 Oct 1 [citado 2015 Oct 23];134(10):123742. Disponible en: http://www.researchgate.net/ publication/246811844_Plipos_vesiculares_correlacin_entr e_hallazgos_ecogrficos_e_histopatolgicos

20. Torres GA, Subiabre DF, Gundelach JI, Aguilera P, Schalper J. Neoplasias Benignas de Vesicula Biliar, un paradigma nacional. Rev Anacem [Internet]. 2009;3(2):414. Disponible en: http://revista.anacem.cl/web/wp-content/uploads/ 2011/04/Neoplasias-Benignas-de-Vesicula-Biliar-unparadigma-nacional..pdf

21. Matos ASB de, Baptista HN, Pinheiro C, Martinho F. Pólipos da vesícula biliar. Como e quando tratar? Rev Assoc Med Bras [Internet]. Associação Médica Brasileira; 2010 [citado 2015 Oct 23];56(3):31821. Disponible en: http://www.scielo.br/ scielo.php?script=sci_arttext\&pid=S0104$42302010000300017 \& \operatorname{lng}=e n \& n r m=$ iso\&tlng=pt

22. Hoyos SI, Sierra EC. Gallbladder polyps. Rev Colomb Cirugía [Internet]. Revista Colombiana de Cirugía; 2010 [citado 2015 Jul 8];25(1):3741. Disponible en: http://www. scielo.org.co/scielo.php?script=sci_arttext\&pid=S2011 . $75822010000100005 \& \operatorname{lng}=e n \& n r m=i s o \notin t \operatorname{lng}=e s$

23. Uribe M, Heinet C, Brito F, Bravo D. Actualización en cancer de vesícula biliar. Rev Clínica Médica las Condes [Internet]. 2013;24(4):63843. Disponible en: http://www. clinicalascondes.cl/Dev_CLC/media/Imagenes/PDF revista médica/2013/4 julio/REV--MED--CLIN.-CONDES---2013;24(4)-WEB.pdf

24. Seguel S G, Fres R E, Frez B M, Pinochet B F, Espinosa S R, Suárez M J. Naturaleza de los pólipos de la vesícula biliar sometidos a colecistectomía. Rev Chil cirugía [Internet] Sociedad de Cirujanos de Chile; 2007 Jun [citado 2015 Jul 8];59(3):20811. Disponible en: http://www.scielo.cl/ scielo.php?script =sci_arttext\&pid=S0718. 40262007000300007\&lng=es\&nrm=iso\&tlng=es

25. Bugosen Tannous M, Tagle Arróspide M, Huerta-Mercado Tenorio J, Scavino Levy Y. Pólipos vesiculares: características cínicas y anatomopatológicas en pacientes colecistectomizados en la Clínica Anglo Americana entre los años 1999-2007. Rev Gastroenterol del Perú [Internet]. Sociedad de Gastroenterología del Perú; 2007 [citado 2015 Jul 8];31(1):327. Disponible en: http://www.scielo.org.pe/ scielo.php?script=sci_arttext\&pid=s 1022 . 51292011000100007\&lng=es\&nrm=iso\&tlng=es

26. Donald G, Sunjaya D, Donahue T, Hines OJ. Polyp on ultrasound: now what? The association between gallbladder polyps and cancer. Am Surg [Internet]. David Geffen Schoo of Medicine at UCLA, Los Angeles, California, USA.: Southeastern Surgical Congress; 2013 Oct;79(10):10058. Disponible en: http://search.ebscohost.com/ login. aspx?direct=true\&db=mnh\&AN=24160788\&lang=es\&sit $\mathrm{e}=$ ehost-live

27. Cairns V, Neal CP, Dennison AR, Garcea G. Risk and Costeffectiveness of Surveillance Followed by Cholecystectomy for Gallbladder Polyps. Arch Surg (Chicago, Ill 1960) [Internet]. United States: American Medical Association; 2012 Dec;147(12):107883. Disponible en: http://search.ebscohost. com/login. aspx?direct=true\&db= mnh\&AN=22911224\&lang=es\&site=ehost-live

28. Tomić D V, Marković ARP, Alempijević TM, Davidović DB, Prsić DR, Vucković MS. Ultrasound diagnosis of gallbladder polyps. Acta Chir lugosl [Internet]. Faculty of Medicine, University of Belgrade, Belgrade, Serbia.: Udruzenje Hirurga Jugoslavije; 2011;58(4):315. Disponible en: http: //search.ebscohost.com/login.aspx?direct=true\&db= mnh\&AN=22519188\&lang=es\&site=ehost-live

29. Ito H, Hann LE, DAngelica M, Allen P, Fong Y, Dematteo RP, et al. Polypoid lesions of the gallbladder: diagnosis and followup. J Am Coll Surg [Internet]. 2009 Apr [citado 2015 Dec 1];208(4):5705. Disponible en: http://www.ncbi. nlm.nih.gov/pubmed/19476792

30. Choi SY, Kim TS, Kim HJ, Park JH, Park D Il, Cho YK, et al. Is it necessary to perform prophylactic cholecystectomy for asymptomatic subjects with gallbladder polyps and gallstones? J Gastroenterol Hepatol [Internet]. Department of Internal Medicine, Kangbuk Samsung Hospital, Sungkyunkwan University College of Medicine, Seoul, Korea.: Blackwell Scientific Publications; 2010 Jun;25(6):1099104. Disponible en: http://search. ebscohost.com / login.aspx?direct =true $\& d b=$ mnh\&AN=20594225\&lang=es\&site=ehost-live

31. Lara Mantilla TA. Correlación entre hallazgos ecográficos e histopatológicos del pólipo vesicular de pacientes colecistectomizados. [Internet]. Universidad Nacional de Trujillo; 2009 [citado 2015 Dec 4]. Disponible en: http: / /dspace.unitru.edu.pe: $8080 / x m l u i / h a n d l e /$ $123456789 / 105$

32. Rodríguez F, Díaz R, Rioseco M, Campaña L. Pólipos vesiculares: Correlación entre hallazgos ecográficos e histopatológicos. Rev Argentina Resid Cirugía [Internet]. 2014;19(2):204. Disponible en: http://www.aamrcg.org.ar/ revista/v19n2/3 Polipo Vesiculares.pdf

\section{Fuentes de Financiamiento}

Este trabajo ha sido financiado por los autores.

\section{Conflicto de interés}

Los autores declaran no presentar ningún conflicto de interés.

\section{Correspondencia:}

Emiliano Contreras Castro

Dirección: Malecón Balta 956. Miraflores, Lima. Clínica Good Hope

Teléfono: 3652300 - 182

Correo electrónico:emilianocc@yahoo.es 